\title{
Молекулярная филогения Наmamelidaceae s.l. на основе анализа нуклеотидных последовательностей ITS ядерной и matK, trnL-F хлоропластной ДНК
}

\section{Molecular phylogeny of the Hamamelidaceae s.l. based on the ITS sequences of nuclear DNA and matK, trnL-F sequences of chloroplast DNA}

\author{
Рослов М. С. \\ Roslov M. S. \\ Московский государственный университет имени М. В. Ломоносова, г. Москва, Россия. E-mail: maxim_roslov@mail.ru
}

Lomonosov Moscow State University, Moscow, Russia

\begin{abstract}
Peфераm. Hamamelidaceae s.1. - семейство, которое служит своеобразным мостом между базальными евдикотами и розидами, поэтому лучшее понимание филогении семейства важно для выявления эволюционных закономерностей в диверсификации евдикот. Межродовые отношения у Наmamelidaceae s.1. являются предметом дискуссий. В ходе работы были реконструированы филогенетические взаимоотношения между 37 видами Нататеlidaceae s.l. и Cercidiphyllum japonicum в качестве внешней группы. Для этого молекулярно-генетические данные (3 маркера: matK, trnL-trnF, ITS-5.8S rRNA) были обработаны методом максимального правдоподобия. Полученная кладограмма показывает, что сем. Наmamelidaceae s.l. полифилетично. Наmamelidaceae s.s. образует монофилетическую группу. На кладограмме выделено пять основных клад: Altingiaceae; Rhodoleioideae sensu Endress вместе c Exbucklandioideae sensu Endress (исключая Disanthus и Mytilaria); Mytilaria; Disanthus; Hamamelidoideae sensu Endress. С небольшими изменениями в Hamamelidoideae поддерживается монофилия нескольких ранее признанных групп, в том числе триб: Corylopsideae sensu Endress, Eustigmateae sensu Endress (включая Molinadendron), Fothergilleae (включая Hamamelis, исключая Molinadendron и Matudaea), а также подтриб Dicoryphinae sensu Endress и Loropetalinae sensu Endress (включая Matudaea).
\end{abstract}

Ключевые слова. Алтингиевые (Altingiaceae), гамамелисовые (Наmamelidaceae), метод максимального правдоподобия, молекулярная филогения, филогенетические взаимоотношения.

Summary. The Hamamelidaceae s.l. is a family that bridges the basal elements of the rosids and the "lower" eudicots, thus a better understanding of the phylogeny of the family is important for clarifying evolutionary patterns in the diversification of eudicots. Intergeneric relationships in the Hamamelidaceae s.l. are controversial. In this study, phylogenetic relationships among the 37 species of Hamamelidaceae s.l. and Cercidiphyllum japonicum as the outgroup were reconstructed using the molecular data (matK, trnL-trnF, ITS-5.8S rRNA sequences) by maximum likelihood criterion. This data indicate that Hamamelidaceae s.l. is polyphyletic. Hamamelidaceae s.s. is monophyletic. Five clades were recognized in the phylogenetic tree: Altingiaceae; Rhodoleioideae sensu Endress, plus Exbucklandioideae sensu Endress (excluding Disanthus and Mytilaria); Mytilaria; Disanthus; Hamamelidoideae sensu Endress. In the Hamamelidoideae, the phylogeny supports the monophyly of several previously recognized groups with modifications, including the tribes Corylopsideae sensu Endress, Eustigmateae sensu Endress (including Molinadendron), Fothergilleae (including Hamamelis, excluding Molinadendron and Matudaea), and the subtribes Dicoryphinae sensu Endress and Loropetalinae sensu Endress (including Matudaea).

Key words. Altingiaceae, Hamamelidaceae, maximum likelihood estimation, molecular phylogeny, phylogenetic relationships.

Семейство гамамелисовые (Наmamelidaceae R. Brown s. 1.) объединяет вечнозеленые или листопадные деревья или кустарники. Листья очередные, реже супротивные, цельные или лопастные, с прилистниками. Жилкование перистое или пальчатое. Соцветия - колосья или головки, реже кисти или метелки, терминальные и пазушные. Цветки мелкие или средние, актиноморфные, редко зигоморфные, 
обое- или однополые. Околоцветник двойной или простой. Чашечка 4-5-членная, иногда отсутствует. Венчик отсутствует или 4-5-членный. Лепестки белые, желтые, зеленоватые, красные, часто лентовидные. Тычинок 4-5, иногда больше. Гинецей синкарпный, карпелл 2. Завязь полунижняя или почти нижняя, изредка верхняя, двухгнездная. Плод - коробочка с кожистым наружным и твердым внутренним слоем (Endress, 1993; Meyer, 1997; Zhang et al., 2003; Stevens, 2017).

Hamamelidaceae s.l. объединяет около 30 родов и более 100 видов растений (Endress, 1993; Meyer, 1997; Zhang et al., 2003; The Plant List, 2013; Stevens, 2017; Christenhusz, Byng, 2019). Ареал дизъюнктивный, включает Западную, Центральную, Восточную и Юго-Восточную Азию, Северную Австралию, Южную и Восточную Африку, Северную Центральную и Южную Америку (Meyer, 1997; Zhang et al., 2003; Shatilovaet al., 2016; Stevens, 2017).

Филогенетические взаимоотношения в пределах Hamamelidaceae s.1. дискуссионны. По результатам сравнительно-морфологического анализа таксон считался монофилетическим (Endress, 1989; 1993; Zhang et al., 2003), однако по данным молекулярной филогенетики группа является парафилетической, на основании чего разделяется на 2 семейства: Altingiaceae Horaninow и Hamamelidaceae s.s. (Angiosperm Phylogeny Group, 1998; 2003; 2009; 2016; The Plant List, 2013; Stevens, 2017). Вопросу изучения систематики данной группы посвящено несколько исследований (Manos, Steele, 1997; Li et al., 1997; 1999a; 1999b; Qiu et al., 1998; Shi et al., 1998; Li et al., 2000; Ickert-Bond, Wen, 2006; Ickert-Bond et al., 2007). Однако в вышеперечисленных работах в анализе участвует лишь часть таксона Hamamelidaceae s.l. (Hamamelis, Corylopsis, Altingiaceae), либо же используются данные только по одному участку ДНК. В данном исследовании проанализированы последовательности 3 участков ядерной и хлоропластной ДНК представителей практически всех родов Hamamelidaceae s.l.

Цель данного исследования - построение модели филогенетических взаимоотношений в пределах Hamamelidaceae s.l. на основе анализа нуклеотидных последовательностей. Для реконструкции филогенетических отношений в пределах группы был использован метод построения филогенетических деревьев на основе кладистического анализа (Hennig, 1950; 1966). В качестве молекулярных данных из базы данных ведущего генетического банка GenBank (Benson et al., 2013) были взяты результаты расшифровки последовательностей 2 участков хлоропластной (matK; trnL-trnF) и 1 участка ядерной ДНК (ITS-5.8S rRNA) 37 видов из 27 родов Наmamelidaceae s.l. (5 видов из 2 родов Altingiaceae и 32 вида из 25 родов Hamamelidaceae s.s.) В качестве внешней группы выбран Cercidiphyllum japonicum Siebold et Zucc. ex J.J.Hoffm. et J.H.Schult.bis. из семейства багрянниковые (Cercidiphyllaceae). Номера доступа избранных последовательностей ДНК в базе данных GenBank представлены в табл.

Полученные нуклеотидные последовательности были выравнены методами MUSCLE (Edgar, 2004) и Clustal Omega (Sievers et al., 2011) с использованием программного обеспечения Unipro UGENE v. 1.30.0 (Okonechnikov et al., 2012). Для редактирования вручную более подходящими оказались выравнивания, полученные с использованием метода Clustal Omega. Отредактированные выравнивания по 3 участкам ДНК объединены в единую матрицу данных, в результате чего было получено множественное выравнивание суммарной длиной 3100 нуклеотидов.

Полученные данные были обработаны в программе MEGA-X (Kumar et al., 2018) методом максимального правдоподобия (Felsenstein, 1981) с бутстрэп-поддержкой 1000 итераций. В результате была построена молекулярная кладограмма (рис. 1).

В ходе анализа полученной кладограммы следует отметить, что Hamamelidaceae s.l. не образует монофилетическую группу, что согласуется с современными представлениями (Angiosperm Phylogeny Group, 1998; 2003; 2009; 2016; The Plant List, 2013; Stevens, 2017). Altingiaceae образует монофилетическую группу, а Наmamelidaceae s. s. - политомию с Cercidiphyllum japonicum, поскольку показатель бутстрэпа в данном случае оказался недостаточен. Однако в соответствии с результатами других исследований (Qiu et al., 1998) Hamamelidaceae s.s. монофилетично.

Altingiaceae соответствует подсемейству Altingioideae Reinsch sensu Endress (1989), но в нем нарушается монофилия родов. Восточноазиатский Liquidambar acalycina H.T.Chang является сестринским к восточноазиатскому виду Altingia obovata Merr. et Chun, а не к североамериканскому Liquidambar styraciflua L. и западноазиатскому Liquidambar orientalis Mill. О несоответствии данных сравнительно-морфологического и молекулярно-генетического анализа в Altingiaceae было известно и ранее 
Номера доступа изученных последовательностей ДНК в базе данных GenBank

\begin{tabular}{|c|c|c|c|}
\hline Вид & matK & $\operatorname{trnL}-\operatorname{trnF}$ & ITS-5.8S rRNA \\
\hline $\begin{array}{l}\text { Cercidiphyllum japonicum Siebold \& Zucc. ex J. J. } \\
\text { Hoffm. et J. H. Schult. bis }\end{array}$ & L11673. & AM397171.1 & HQ652480.1 \\
\hline Altingia excelsa Noronha & DQ352374.1 & DQ352225.1 & AF304525.1 \\
\hline Altingia obovata Merr. et Chun & DQ352377.1 & DQ352204.1 & AF133234.1 \\
\hline Liquidambar acalycina $\mathrm{H}$. T. Chang & DQ352380.1 & DQ352216.1 & AF133231.1 \\
\hline Liquidambar orientalis Mill. & DQ352383.1 & DQ352222.1 & AF133229.1 \\
\hline Liquidambar styraciflua L. & DQ352381.1 & DQ352217.1 & EU646183.1 \\
\hline Chunia bucklandioides H. T. Chang & AF108466.1 & EF456729.1 & AF162211.1 \\
\hline Corylopsis glabrescens Hand.-Mazz. & AB236998.1 & GU576816.1 & GU576645.1 \\
\hline Corylopsis pauciflora Siebold et Zucc. & $\mathrm{AB} 236991.1$ & AF147465.1 & AB299314.1 \\
\hline Dicoryphe stipulacea J. St.-Hil. & AF013040.1 & AF147466.1 & GU576646.1 \\
\hline Disanthus cercidifolius Maxim. & AF081069.1 & DQ352199.1 & AF127507.1 \\
\hline Distyliopsis tutcheri (J.H.Hemsl.) Endress & AF013042.1 & AF147470.1 & AH005557.2 \\
\hline Distylium myricoides Hemsl. & GU576683.1 & AF147468.1 & GU576648.1 \\
\hline Distylium racemosum Siebold et Zucc. & AF013041.1 & AF147469.1 & AF074249.1 \\
\hline Eustigma oblongifolium Gardner et Champ. & AF013043.1 & AF147471.1 & AF127518.1 \\
\hline Exbucklandia populnea (R.Br. ex Griff.) R.W.Br. & AF128831.1 & AF147472.1 & GU576649.1 \\
\hline Exbucklandia tonkinensis (Lecomte) H.T.Chang & AF128832.1 & DQ352198.1 & GU576650.1 \\
\hline Fortunearia sinensis Rehder et E.H.Wilson & AF013044.1 & AF147473.1 & AF127515.1 \\
\hline Fothergilla gardenii $\mathrm{L}$. & KT438384.1 & KT438462.1 & GU576651.1 \\
\hline Fothergilla major Lodd. & AF013045.1 & AF147474.1 & AF074250.2 \\
\hline Hamamelis mollis Oliv. ex F. B. Forbes et Hemsl. & AF248619.1 & DQ352357.1 & GU576657.1 \\
\hline Hamamelis virginiana $\mathrm{L}$. & AF013046.1 & DQ352196.1 & GU576666.1 \\
\hline Loropetalum chinense (R. Br.) Oliv. & AF013059.1 & AF147476.1 & GU576672.1 \\
\hline Loropetalum subcordatum (Benth.) Oliv. & MG457805.1 & MG457805.1 & AF022242.1 \\
\hline Maingaya malayana Oliv. & AF025393.1 & AF147477.1 & AF022241.1 \\
\hline Matudaea trinervia Lundell & AF013048.1 & AF147478.1 & AF015437.1 \\
\hline $\begin{array}{l}\text { Molinadendron guatemalense (Radlk. ex Harms) } \\
\text { P.K.Endress }\end{array}$ & AF013049.1 & AF147479.1 & AF015438.1 \\
\hline Mytilaria laosensis Lecomte & AF128828.1 & EF456731.1 & GU576673.1 \\
\hline Neostrearia fleckeri L.S.Sm. & AF013050.1 & AF147480.1 & AF015439.1 \\
\hline $\begin{array}{l}\text { Noahdendron nicholasii P. K. Endress, B. Hyland et } \\
\text { Tracey }\end{array}$ & AF013051.1 & AF147481.1 & AF015440.1 \\
\hline Ostrearia australiana Baill. & AF013052.1 & AF147482.1 & AF015441.1 \\
\hline $\begin{array}{l}\text { Parrotia subaequalis (Hung T. Chang) R. M. Hao et H.T. } \\
\text { Wei }\end{array}$ & MG252374.1 & GU576845.1 & GU576674.1 \\
\hline Parrotiopsis jacquemontiana (Decne.) Rehder & AF013054.1 & AF147484.1 & AF015442.1 \\
\hline Rhodoleia championii Hook. f. & AF128833.1 & AF147485.1 & GU576675.1 \\
\hline Rhodoleia henryi Tong & GU576711.1 & GU576847.1 & GU576676.1 \\
\hline Sinowilsonia henryi Hemsl. & AF013056.1 & AF147487.1 & AF127512.1 \\
\hline Sycopsis sinensis Oliv. & AF013057.1 & AF147488.1 & GU576677.1 \\
\hline Trichocladus crinitus Pers. & AF013058.1 & AF147490.1 & AF147755.1 \\
\hline
\end{tabular}

(Ickert-Bond, Pigg, Wen, 2007). Филогенетическая близость восточноазиатских видов родов Altingia Nor. и Liquidambar L. хорошо согласуется с биогеографией (Ickert-Bond, Wen, 2006). Таким образом, для сохранения монофилии род Altingia должен быть погружен в род Liquidambar.

B Hamamelidaceae s. s. на кладограмме выделяются клады, по составу родов соответствующие подсемействам Rhodoleioideae Harms sensu Endress (1989); Exbucklandioideae Reinsch sensu Endress (1989), исключая Disanthus Maxim. и Mytilaria Lecomte, и Hamamelidoideae Reinsch sensu Endress (1989). 


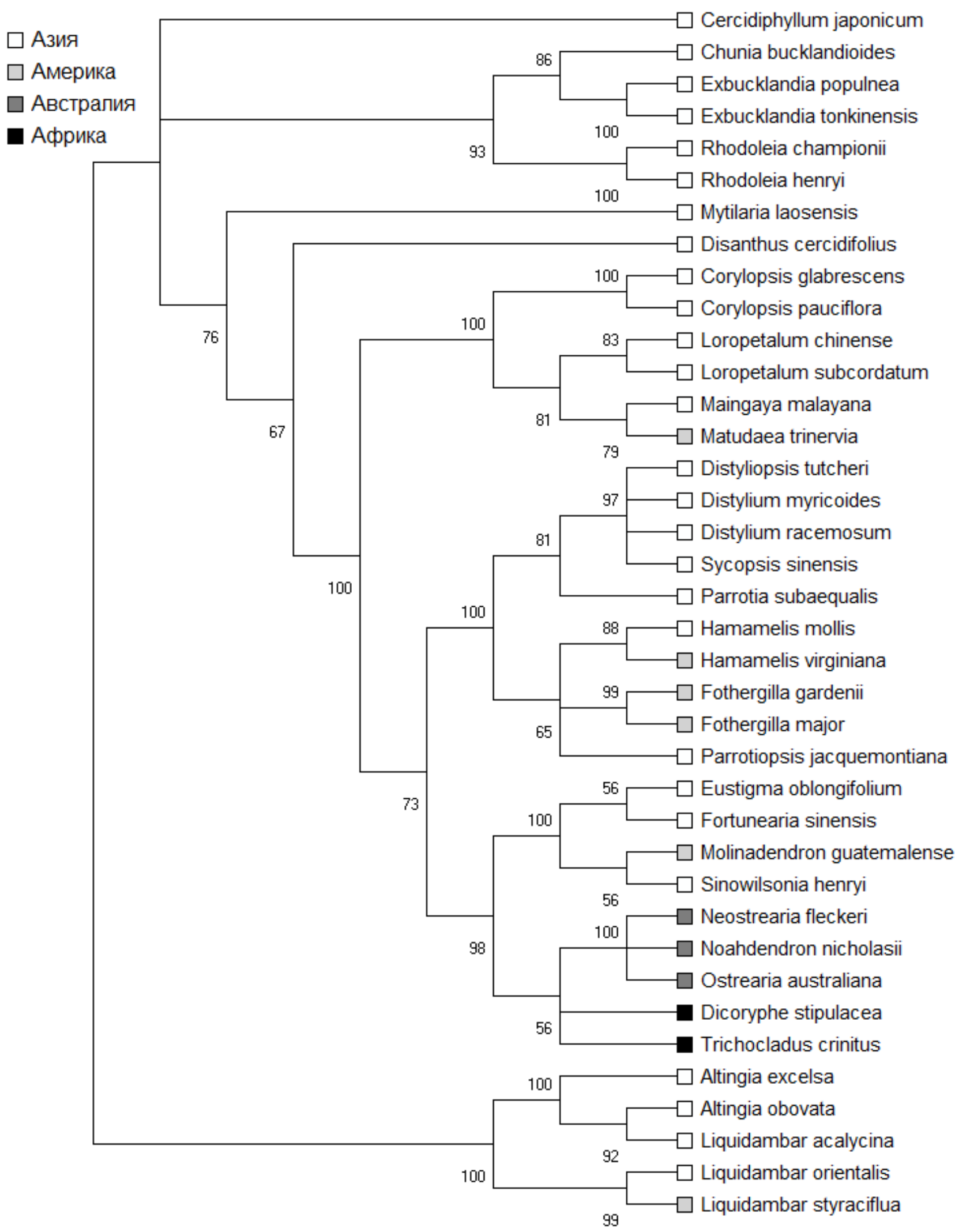

Рис. 1. Молекулярная кладограмма Наmamelidaceae s. 1., построенная на основе анализа нуклеотидных последовательностей matK, trnL-trnF, ITS-5.8S rRNA методом максимального правдоподобия в программе MEGA-X. Условные обозначения: квадратами на концах ветвей показаны области распространения анализируемых видов; в узлах цифрами показан показатель бутстрэпа, величины $<50$ не отображены. 
Disanthus и Mytilaria являются сестринскими группами к кладам Hamamelidoideae и HamamelidoideaeDisanthus, соответственно.

В подсемействе Hamamelidoideae выделяются клады, соответствующие трибам Corylopsideae Harms sensu Endress (1989); Eustigmateae Harms sensu Endress (1989), включая Molinadendron Endress, и Fothergilleae A. DC. sensu Endress (1989), включая Hamamelis L., исключая Molinadendron, Matudaea Lundell. Триба Наmamelideae A. DC. sensu Endress (1989) является полифилетичной. Она разделяется на 2 монофилетических клады, соответствующие субтрибам Loropetalinae Endress (1989), включая Matudaea, и Dicoryphinae Endress (1989).

Таким образом, следует констатировать, что для Наmamelidaceae s. 1. филогения, основанная на анализе молекулярно-генетических данных, отличается от таковой, основанной на сравнительно-морфологическом анализе. В дальнейшем для большей репрезентативности может быть перспективным увеличение числа анализируемых видов и участков ДНК. Это могло бы уменьшить число политомий и увеличить поддержку ключевых узлов. В свою очередь, данные о филогении открывают возможность реконструировать историю расселения таксонов (van Veller et al., 2003; Arias, 2011).

\section{ЛИТЕРАТУРА}

Angiosperm Phylogeny Group. An ordinal classification for the families of flowering plants // Missouri Botanical Garden Press Annals of the Missouri Botanical Garden, 1998. - Vol. 85, №. 4. - P. 531-553.

Angiosperm Phylogeny Group. An update of the Angiosperm Phylogeny Group classification for the orders and families of flowering plants: APG II // Botanical Journal of the Linnean Society, 2003. - Vol. 141, №. 4. - P. 399-436.

Angiosperm Phylogeny Group. An update of the Angiosperm Phylogeny Group classification for the orders and families of flowering plants: APG III // Botanical Journal of the Linnean Society, 2009. - Vol. 161, №. 2. - P. 105-121.

Angiosperm Phylogeny Group. An update of the Angiosperm Phylogeny Group classification for the orders and families of flowering plants: APG IV // Botanical Journal of the Linnean Society, 2016. - Vol. 181, №. 1. - P. 1-20.

Arias $\boldsymbol{J}$. S. A primer in phylogenetic biogeography using the spatial analysis of vicariance. 2011. $-31 \mathrm{p}$

Benson D. A., Clark K., Karsch-Mizrachi I., Lipman D. J., Ostell, J., Sayers E. W. GenBank // Nucleic Acids Research, 2013. - Vol. 42, № D1. - P. D32-D37.

Christenhusz M. J. M., Byng J. W. The number of known plants species in the world and its annual increase // Phytotaxa, 2016. - Vol. 261, №. 3. - P. 201-217.

Edgar R. C. MUSCLE: multiple sequence alignment with high accuracy and high throughput // Nucleic Acids Research, 2004. - Vol. 32, №. 5. - P. 1792-1797.

Endress P. K. A suprageneric taxonomic classification of the Hamamelidaceae // Taxon, 1989. - Vol. 38, №. 3. P. 371-376.

Endress P. K. Hamamelidaceae // The families and genera of vascular plants. Vol. 2. Flowering Plants. Dicotyledons: Magnoliid, Hamamelid and Caryophyllid Families. - Berlin: Springer-Verlag, 1993. - P. 322-331.

Felsenstein J. Evolutionary trees from DNA sequences: a maximum likelihood approach // Journal of Molecular Evolution, 1981. - Vol. 17, №. 6. - P. 368-376.

GenBank. An annotated collection of all publicly available DNA sequences. URL: https://www.ncbi.nlm.nih.gov/ genbank/ (Accessed 15 November 2018).

Hennig $\boldsymbol{W}$. Grundzüge einer Theorie der Phylogenetischen Systematik. - Berlin: Deutscher Zentralverlag, 1950. $370 \mathrm{~s}$.

Hennig $\boldsymbol{W}$. Phylogenetic systematics. - Urbana: University of Illinois Press, 1966. - 263 p.

Ickert-Bond S. M., Pigg K. B., Wen J. Comparative infructescence morphology in Altingia (Altingiaceae) and discordance between morphological and molecular phylogenies // American Journal of Botany, 2007. - Vol. 94, №. 7. - P. 1094-1115.

Ickert-Bond S. M., Wen J. Phylogeny and biogeography of Altingiaceae: evidence from combined analysis of five non-coding chloroplast regions // Molecular Phylogenetics and Evolution, 2006. - Vol. 39, №. 2. - P. 512-528.

Kumar S., Stecher G., Li M., Knyaz C., Tamura K. MEGA X: Molecular Evolutionary Genetics Analysis across computing platforms // Molecular Biology and Evolution, 2018. - Vol. 35, №. 6. - P. 1547-1549.

Li J., Bogle A. L., Klein A. S. Phylogenetic relationships in the Corylopsis complex (Hamamelidaceae): evidence from sequences of the internal transcribed spacers of nuclear ribosomal DNA and morphology // Rhodora, 1997. - Vol. 99. - P. 302-318.

Li J., Bogle A. L., Klein A. S. Phylogenetic relationships in the Hamamelidaceae: Evidence from the nucleotide sequences of the plastid gene matK // Plant Systematics and Evolution, 1999a. - Vol. 218, №. 3-4. - P. 205-219. 
Li J., Bogle A. L., Klein A. S. Phylogenetic relationships of the Hamamelidaceae inferred from sequences of internal transcribed spacers (ITS) of nuclear ribosomal DNA // American Journal of Botany, 1999b. - Vol. 86, №. 7. - P. 10271037.

Li J., Bogle A. L., Klein A. S., Donoghue M. J. Phylogeny and biogeography of Hamamelis (Hamamelidaceae) // Harvard Papers in Botany, 2000. - Vol. 5, №. 1. - P. 171-178.

Manos P. S., Steele K. P. Phylogenetic analyses of "higher" Hamamelididae based on plastid sequence data // American Journal of Botany, 1997. - Vol. 84, №. 10. - P. 1407-1419.

Meyer F. G. Hamamelidaceae // Flora of North America North of Mexico. Vol. 3. Magnoliophyta: Magnoliidae and Hamamelidae. - New York: Oxford University Press, 1997. - P. 358-367.

Okonechnikov K., Golosova O., Fursov M. Unipro UGENE: a unified bioinformatics toolkit // Bioinformatics, 2012. - Vol. 28, №. 8. - P. 1166-1167.

Qiu Y. L., Chase M. W., Hoot S. B., Conti E. Phylogenetics of the Hamamelidae and their allies: parsimony analyses of nucleotide sequences of the plastid gene rbcL // International Journal of Plant Sciences, 1998. - Vol. 159, №. 6. - P. 891-905.

Shatilova I., Rukhadze L., Kokolashvili I. Representatives of the family Hamamelidaceae in Neogene of Georgia. Tbilisi: Georgian National Museum, 2016. - 80 p.

Shi S., Chang H. T., Chen Y., Qu L., Wen J. Phylogeny of the Hamamelidaceae based on the ITS sequences of nuclear ribosomal DNA // Biochemical Systematics and Ecology, 1998. - Vol. 26, №. 1. - P. 55-69.

Sievers F., Wilm A., Dineen D., Gibson T. J., Karplus K., Li W., Lopez R., McWilliam H., Remmert M., Söding J., Thompson J. D., Higgins D. G. Fast, scalable generation of high-quality protein multiple sequence alignments using Clustal Omega // Molecular Systems Biology, 2011. - Vol. 7, №. 1. - P. 539.

Stevens P. F. 2017. Angiosperm Phylogeny Website. Version 14. URL: http://www.mobot.org/MOBOT/research/APWeb/ (Accessed 02 February 2019).

The Plant List. Version 1.1. 2013. A working list of all known plant species. URL: http://www.theplantlist.org/ (Accessed 02 February 2019).

van Veller M. G. P., Brooks D. R., Zandee M. Cladistic and phylogenetic biogeography: the art and the science of discovery // Journal of Biogeography, 2003. - Vol. 30, №. 3. - P. 319-329.

Zhang Z., Zhang H., Endress P. K. Hamamelidaceae // Flora of China. Vol. 9. Pittosporaceae through Connaraceae. Beijing and St. Louis: Science Press and Missouri Botanical Garden, 2003. - P. 18-42. 